\title{
Self-starting lithium niobate soliton microcombs
}

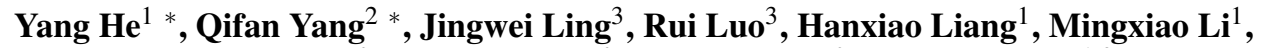 \\ Boqiang Shen ${ }^{2}$, Heming Wang ${ }^{2}$, Kerry Vahala ${ }^{2}$, and Qiang Lin ${ }^{1,3}$ \\ ${ }^{1}$ Department of Electrical and Computer Engineering, University of Rochester, Rochester, New York 14627, USA \\ ${ }^{2}$ T. J. Watson Laboratory of Applied Physics, California Institute of Technology, Pasadena, California 91125, USA \\ ${ }^{3}$ Institute of Optics, University of Rochester, Rochester, New York 14627, USA \\ *These authors contributed equally to this work
}

\begin{abstract}
We report soliton generation in a high-Q lithium niobate resonator. The photorefractive effect enables self-starting mode locking and is able to produce stable single solitons on demand that feature reversible switching between soliton states. ( 2019 The Author(s)
\end{abstract}

OCIS codes: (130.3730) Lithium niobate; (130.3990) Micro-optical devices.

The recent demonstration of soliton mode locking in microresonators [1] represents a major turning point in the subject of frequency microcombs and many material systems and cavity geometries are being explored for various applications [2]. In this work soliton generation in a high-Q lithium niobate (LN) resonator is observed for the first time. Moreover, on account of the intriging properties of lithium niobate the soliton mode locked system is able to self-start. Specifically, soliton microcombs must be pumped at a frequency that is red detuned relative to a cavity resonance [2], but this regime is also unstable due to a thermo-optical nonlinearity [3]. As a result special techniques for pumping and triggering solitons have been developed [2]. Here the photorefractive property of LN is shown to allow stable operation and pumping on the red-detuned side of resonance. As a result, self-starting mode locking of soliton microcombs is demonstrated by a simple and reversible pump tuning process.
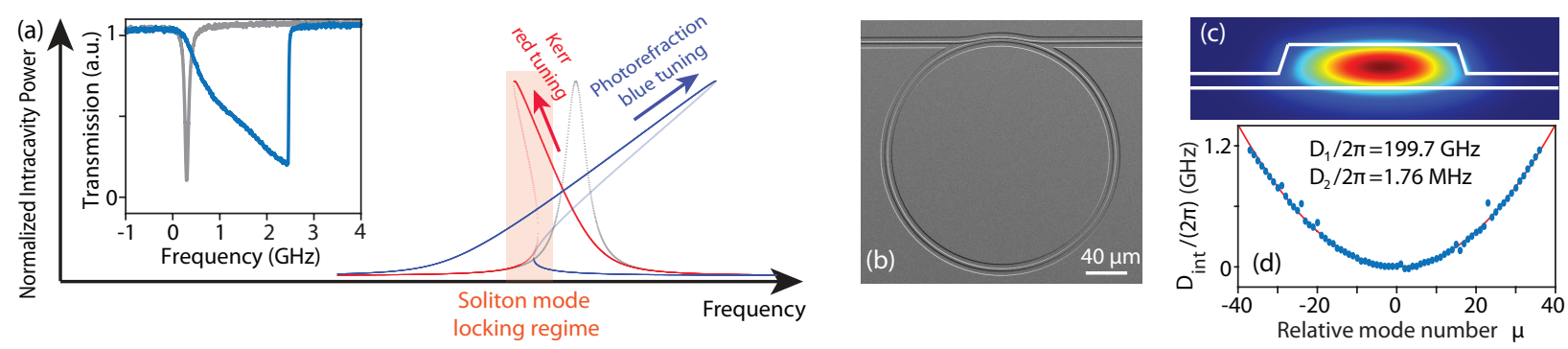

Fig. 1. (a) Schematic showing the resonance tuning induced by different mechanisms. The gray curve shows the Lorentzian-shaped intrinsic cavity resonance. The inset shows laser-scanned transmission spectra of a quasiTE cavity mode, with input powers of $2 \mu \mathrm{W}$ (grey) and $0.7 \mathrm{~mW}$ (blue). (b) SEM image of a LN microring resonator. (c) Simulated optical mode profile of the fundamental cavity mode. (d) Recorded dispersion of the soliton forming mode family as a function of the relative mode number $\mu$, where $D_{\text {int }}=\omega_{\mu}-\omega_{0}-\mu D_{1}$ and $\mu=0$ is around $1558.7 \mathrm{~nm}$. Experimental data are shown as blue dots and a theoretical fitting is shown in red.

LN features a strong photorefractive effect, which causes an intensity-dependent decrease of refractive index [4]. Moreover, LN exhibits a negligible thermo-optic coefficient for the ordinary polarized light (around room temperature) [5], leading to a suppressed thermo-optic nonlinearity. The combination of these two effects results in a net decrease of refractive index with increased optical intensity. The inset of Fig. 1(a) shows a clear example where the introduced resonance blueshift leads to a triangular-like shape of cavity transmission that faces towards higher frequency. This behavior is opposite to that induced by thermo-optic and/or optical Kerr nonlinearities in conventional Kerr soliton microresonators [2]. As shown in Fig. 1(a), the optical Kerr effect from the soliton shifts the resonance towards the red (red curve), while the photorefractive effect significantly shifts the resonance towards the blue (blue curve). As a result, the soliton formation regime (shaded region) resides directly within the laser detuning regime that is self stabilized by the photorefractive effect, thereby enabling self-starting soliton mode locking.

To show this capability, we used a LN microresonator (Fig. 1(b)(c)) which exhibits a loaded optical Q of $2.2 \times 10^{6}$ and has a radius of $100 \mu \mathrm{m}$. The group velocity dispersion of the device is engineered to be slightly anomalous. The 
dispersion of the mode family is described by the Taylor expansion of the cavity resonance frequency $\omega_{\mu}$ around a reference resonance $\omega_{0}$ [6], $\omega_{\mu}=\omega_{0}+\mu D_{1}+\frac{1}{2} \mu^{2} D_{2}+\frac{1}{6} \mu^{3} D_{3}+\cdots$, shown as Fig. 1(d), with $\beta_{2}=-0.047 \mathrm{ps}^{2} / \mathrm{m}$ $\left(D_{1} /(2 \pi)=199.7 \mathrm{GHz}\right.$ and $\left.D_{2} /(2 \pi)=1.76 \mathrm{MHz}\right)$.
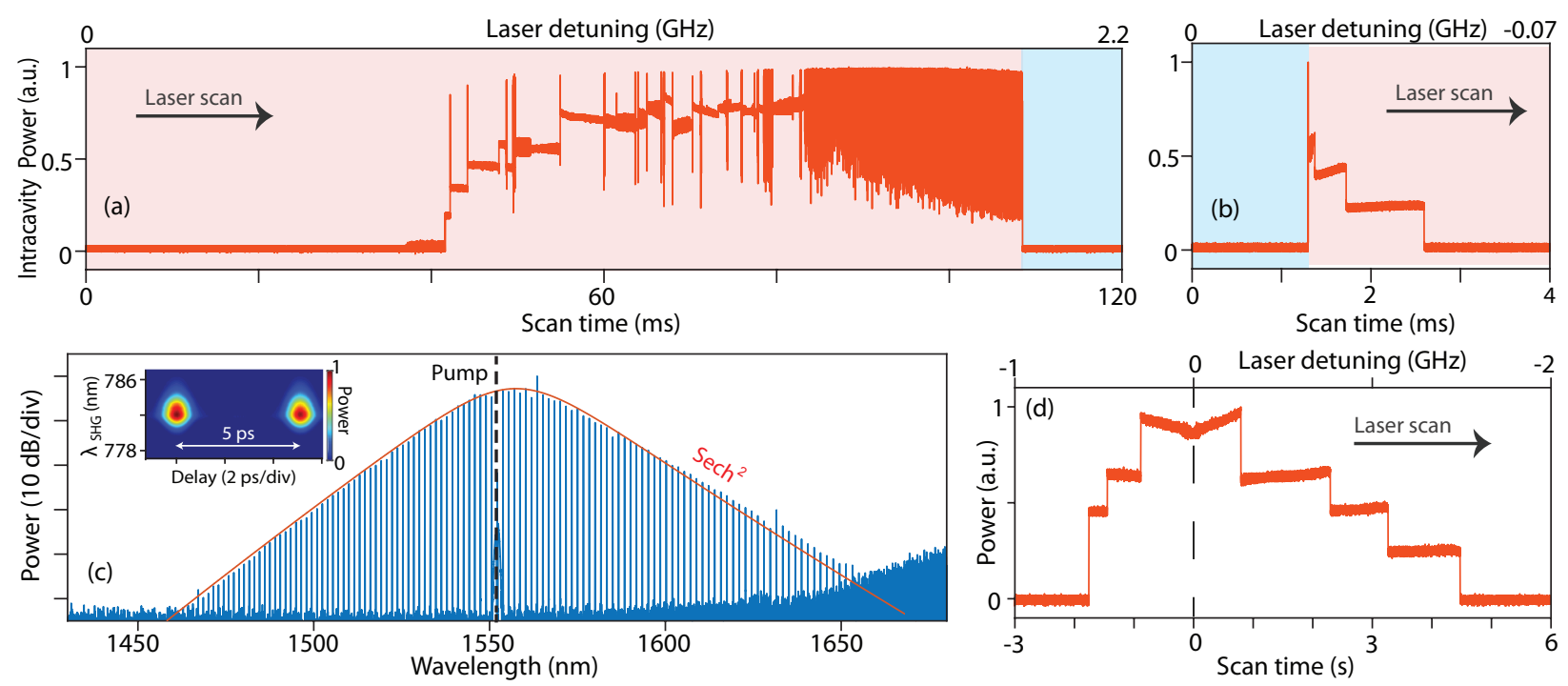

Fig. 2. (a)-(b) Intracavity power as a function of time when the laser is scanned from red to blue (long to short wavelength) (a) and blue to red (b) across the pump resonance. (c) Optical spectrum of the single soliton state. The black dotted line indicates the pump mode. The inset shows a FROG trace. (d) Experimental trace of forward tuning and backward tuning of the pump frequency.

To produce Kerr combs, a pump power of $33 \mathrm{~mW}$ is coupled onto the chip. When the pump frequency is scanned into a cavity resonance from long wavelength (red-detuned side), the average intracavity power readily shows clear discrete steps (Fig. 2(a)). Figure 2(c) shows the spectrum mearsured for the single soliton at the first power step, which exhibits a smooth $\operatorname{sech}^{2}$-shaped spectral envelope. To characterize the short pulse temporal waveform of the solitons, frequency-resolved optical gating (FROG) was used (inset of Fig. 2(c)). The recorded FROG spectrogram shows coherent pulse waveforms with a repetition rate of $5 \mathrm{ps}$, corresponding to the mode spacing of the microcomb. The LN system has another unique and important property in that it can reversibly switch the soliton number up-anddown by tuning the pump wavelength. In Fig. 2(d) the system was first tuned to begin soliton generation and then the pump was swept forward (from long to short wavelength) or backward (from short to long wavelength) to change the soliton number.

In summary, we report a Kerr soliton microcomb system in lithium niobate that is able to self-start by smoothly tuning from the red side of a pumping resonance. The unique features of the device also enable reversible switching of multi-soliton states (back and forth) on demand.

\section{References}

1. T. Herr, et al, "Temporal solitons in optical microresonators," Nature Photon. 8, 145-152 (2014).

2. T. J. Kippenberg, A. L. Gaeta, M. Lipson, and M. L. Gorodetsky, "Dissipative Kerr solitons in optical microresonators," Science 361, 567 (2018).

3. T. Carmon, L. Yang, K J Vahala, et al, "Dynamical thermal behavior and thermal self-stability of microcavities," Optics Express 12, 4742-4750 (2004).

4. P. Günter and J.-P. Huignard, eds., Photorefractive Materials and Their Applications 1, 2 (Springer, New York, 2006).

5. L Moretti, M. Lodice, F. G. D. Corte, and I. Rendina, "Temperature dependence of the thermo-optic coefficient of lithium niobate, from 300 to $515 \mathrm{~K}$ in the visible and infrared regions," J. Appl. Phys. 98, 036101 (2005).

6. X. Yi, et al, "Soliton frequency comb at microwave rates in a high-Q silica microresonator," Optica 2, 1078-1085 (2015) 